\title{
Evolution morphologique d'une plage macrotidale à barres à l'échelle saisonnière
}

\author{
Stepanian A. (Doctorant),Levoy F. (MdC), et Larsonneur C. (Pr.) \\ Unité M2C "Morphodynamique Continentale et Côtière», UPRES-A \\ CNRS 6143 - Université de CAEN. 24 rue des Tilleuls, 14000 CAEN
}

Résumé : Les évolutions morphologiques mensuelles observées sur une plage macrotidale à barres (Normandie) sont mises en relation avec les caractéristiques de l'agitation incidente. La mesure des variations du volume sableux de la plage suggère que les évolutions s'opèrent sans perte sédimentaire significative. Une accrétion des barres et une migration vers le haut estran sont constatées en période de houles faibles, alors que suite à une tempête, une destruction des barres de haute plage est observée.

Abstract : A monthly topographic survey of a macrotidal ridge and runnel beach (Normandy) during 1999 shows a relation between morphological evolution and incident hydrodynamic conditions. Sand volumes measurements point out a stability of the sediment budget and suggest that no significant sedimentary loss occurs during the survey period. For moderate climate wave, the bar crests grow up and migrate landward, contrary to a post-storm period, where a destruction of upper beach bars is observed.

\section{Introduction}

Un des principaux objectifs du Programme National «Environnement Côtier » (PNEC), chantier " Dynamique Sédimentaire », est l'établissement d'une base de données morphologiques et hydrodynamiques concernant trois plages situées sur les côtes françaises métropolitaines et soumises à des conditions hydrodynamiques contrastées (Manche, Atlantique et Méditerranée). Dans ce cadre, la plage d'Omaha-Beach sur la côte du Calvados (figure 1) fait l'objet d'un suivi mensuel concernant la topographie et les conditions d'agitation incidente depuis le début de l'année 1999.

Cette plage représentative des plages à «barres et bâches 》 du Nord-Ouest européen, se situe dans un contexte hydrodynamique caractérisé par un marnage important et une agitation de type « mer de vent ». La zone intertidale présente de façon quasi-permanente plusieurs barres sableuses, parallèles au trait de côte, incisées par des chenaux d'écoulement. Cette morphologie, conforme à la définition de «Ridges and Runnels » (King et Williams, 1949), est caractéristique d'environnements où plusieurs facteurs sont réunis. La plage d'Omaha-Beach avec une pente moyenne de $1,5 \%$, des sables dont la médiane est comprise entre $185 \mu \mathrm{m}$ et $230 \mu \mathrm{m}$, une agitation d'amplitude modérée en raison du fetch limité en Manche, et un marnage variant de $3.3 \mathrm{~m}$ en périodes de mortes-eaux à $6.1 \mathrm{~m}$ en 
périodes de vives-eaux, présente toutes les conditions caractéristiques des systèmes de barres et bâches (Orford et Wright, 1978).

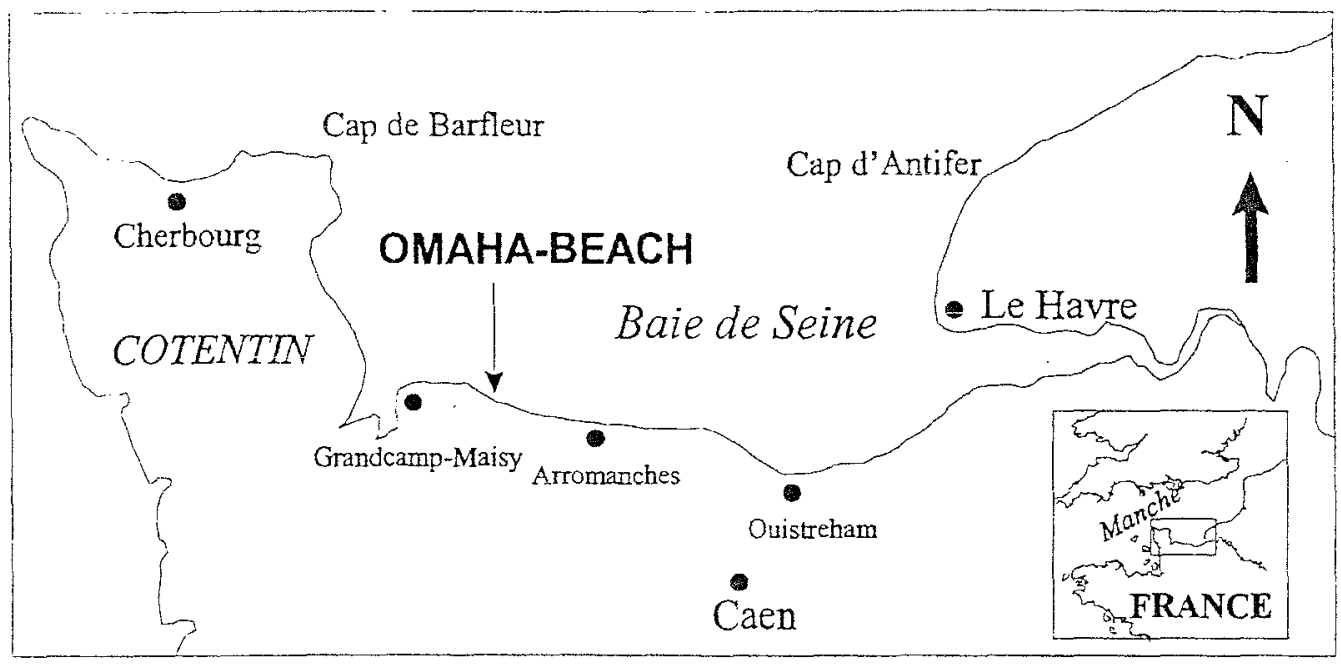

Figure 1 - Localisation de la zone d'étude

Figure I - Location of the study area

Les études entreprises ont pour objectif de préciser les séquences d'évolution morphologique décrivant la mobilité de ces systèmes au travers de l'étude des processus de formation et de destruction des barres à plusieurs échelles de temps. L'approche présentée dans cette note concerne les évolutions morphologiques à un pas de temps mensuel entre février 1999 et novembre 1999.

\section{Méthodologie mises en oeuvre}

\subsection{Levés Topographiques}

Un levé topographique de la plage est effectué mensuellement en marée de vive-eau à l'aide d'un DGPS centimétrique tracté sur la plage, suivant une maille régulière à marée basse. Environ 45 radiales espacées de $10 \mathrm{~m}$ sont réalisées transversalement aux barres sableuses, disposées parallèlement au trait de côte (N110). Le semis de points est traité pour transformer les coordonnées géographiques en coordonnées relatives locales. Un modèle numérique de terrain est obtenu par l'emploi d'un logiciel d'interpolation et de création de blocdiagrammes en 3 dimensions. L'utilisation d'un DGPS permet d'obtenir un semis de points très dense (environ 3000 points) sur une grande surface $(330 \times 410 \mathrm{~m})$ permettant la comparaison de l'état morphologique de la plage d'un mois sur l'autre. Les modèles numériques réalisés sont utilisés pour extraire des profils de plage interpolés et élaborer des cartes d'évolution différentielle, qui traduisent les variations morphologiques 3D subies par la plage entre deux levés.

\subsection{Mesures hydrodynamiques}




\subsection{Mesures hydrodynamiques}

L'évolution morphologique de la plage est fonction des conditions hydrodynamiques entre deux levés. L'acquisition de données concernant l'agitation est donc nécessaire pour mettre en évidence les facteurs à l'origine des évolutions topographiques constatées. Pour cela, un houlographe directionnel S4DW est en permanence installé en bas de plage, au niveau des basses mers de vives-eaux. L'enregistrement des variations de la surface libre et des vitesses orbitales se fait par bursts (séquences d'acquisition à $2 h z$ ) de 9 minutes toutes les 4 heures. Les données brutes obtenues permettent de calculer les caractéristiques de l'agitation incidente ( $\mathrm{Hs}, \mathrm{Tp}, \mathrm{Ts}$, angle d'incidence). En raison des variations du plan d'eau statique avec le cycle tidal, un filtre est appliqué sur les données pour ne retenir que les bursts pendant lesquels la hauteur d'eau moyenne au dessus du capteur est supérieure à $1,5 \mathrm{~m}$.

\section{Variations morphologiques depuis le début de l'année 1999}

Les variations morphologiques mises en évidence, depuis le début des levés, traduisent une évolution du système étudié qui s'est effectuée au cours de trois phases distinctes.

\subsection{Individualisation et migration des barres}

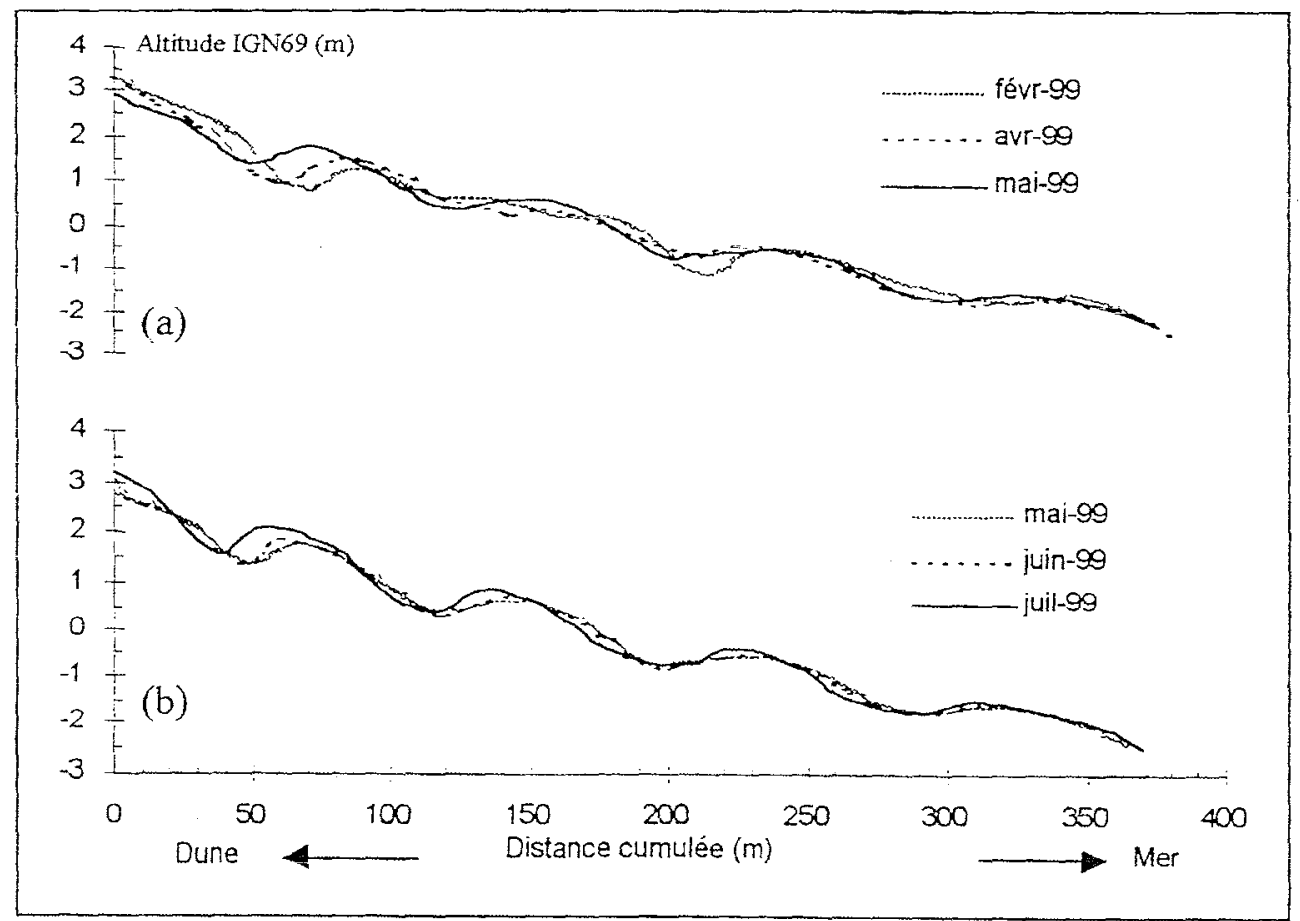

Figure 2 - Evolution du profil central de février à juillet 1999

(a) période de février à mai 1999 (b) période de mai à juillet 1999

Figure 2 - Evolution of the main crosshore profile from February to July 1999

(a) from February to May 1999 (b) from Moy to July 1999 
Entre février et juillet 1999, l'évolution morphologique de la plage est marquée par un exhaussement des barres qui s'individualisent et qui migrent vers le haut de plage comme le présente l'évolution du profil topographique positionné au centre de la zone d'étude (figure 2).

De février à mai 1999 (figure 2a), l'individualisation des barres constitue l'évolution morphologique majeure. Celle-ci est couplée à une migration vers le haut de plage des crêtes de barres et de leur bâche associée. L'individualisation est la conséquence de l'accrétion verticale des crêtes qui atteint $50 \mathrm{~cm}$ pour la première barre sur le haut estran. La migration est particulièrement significative pour les deux barres situées en haute plage, où le déplacement atteint $15 \mathrm{~m}$ vers le haut de plage. Les barres de la basse plage sont à la fois moins mobiles dans le profil et altimétriquement plus stables : la migration de la crête de la barre de bas estran atteint $20 \mathrm{~m}$ en direction du haut de plage, mais l'accrétion verticale est nulle.

De mai à juillet 1999 , le profil conserve la même configuration avec quatre barres distinctes (figure $2 b$ ). la migration des formes présente une cinématique proche de celle observée précédemment. Les crêtes des barres se déplacent respectivement de $15 \mathrm{~m}$ et $10 \mathrm{~m}$ pour les deux premières barres sur la haute plage, alors que l'accrétion verticale est de $50 \mathrm{~cm}$ et $20 \mathrm{~cm}$. Parallèlement à cette migration, leur modelé devient plus asymétrique avec une augmentation significative de la pente 《 onshore » ( $+2 \%$ pour la première barre). Les barres du bas de plage sont, quant à elles, plus stables et subissent une migration des crêtes vers le haut estran moins prononcée, ainsi qu'une faible accrétion (quelques centimètres).

\subsection{Creusement et migration latérale des chenaux de drainage des} bâches

Durant la période estivale entre juillet et septembre 1999, la plage a conservé sa morphologie avec quatre barres bien marquées, mais elle a évolué consécutivement à la migration, parallèlement au trait de côte, de la position des chenaux de drainage des bâches. En effet, apparu nettement en mai, ce système des chenaux de vidange est positionné au mois de juillet 1999 dans l'axe de la radiale 300 (figure 3), avec un exutoire dans la bâche externe au niveau de la radiale 250 . En septembre, ces chenaux se retrouvent positionnés dans l'axe de la radiale 250 avec un exutoire au niveau de la radiale 200 . Une migration "longshore» des chenaux incisant les barres, de $50 \mathrm{~m}$ vers l'Ouest, est mise en évidence.

La comparaison de la position des isolignes $0,1 \mathrm{~m}$ et $-0,7 \mathrm{~m}$ traduit également cette migration latérale vers l'Ouest du système de chenaux d'évacuation (figure 3). 


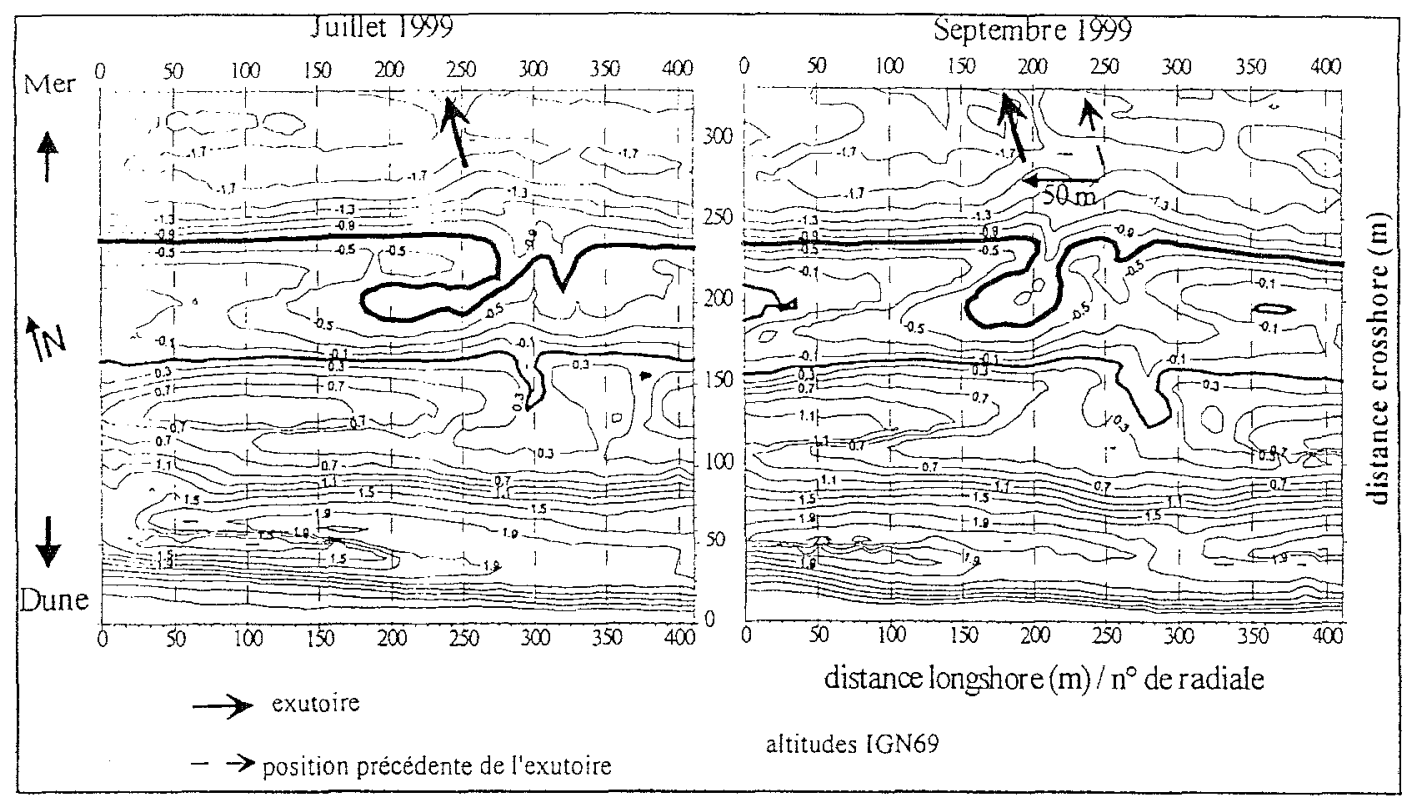

Figure 3 - Topographies de la zone intertidale

Figure 3 - Intertidal beach topographies

\subsection{Destruction des barres de haute plage}

Entre septembre et novembre 1999, la topographie de la plage d'OmahaBeach évolue de manière importante (figure 4).

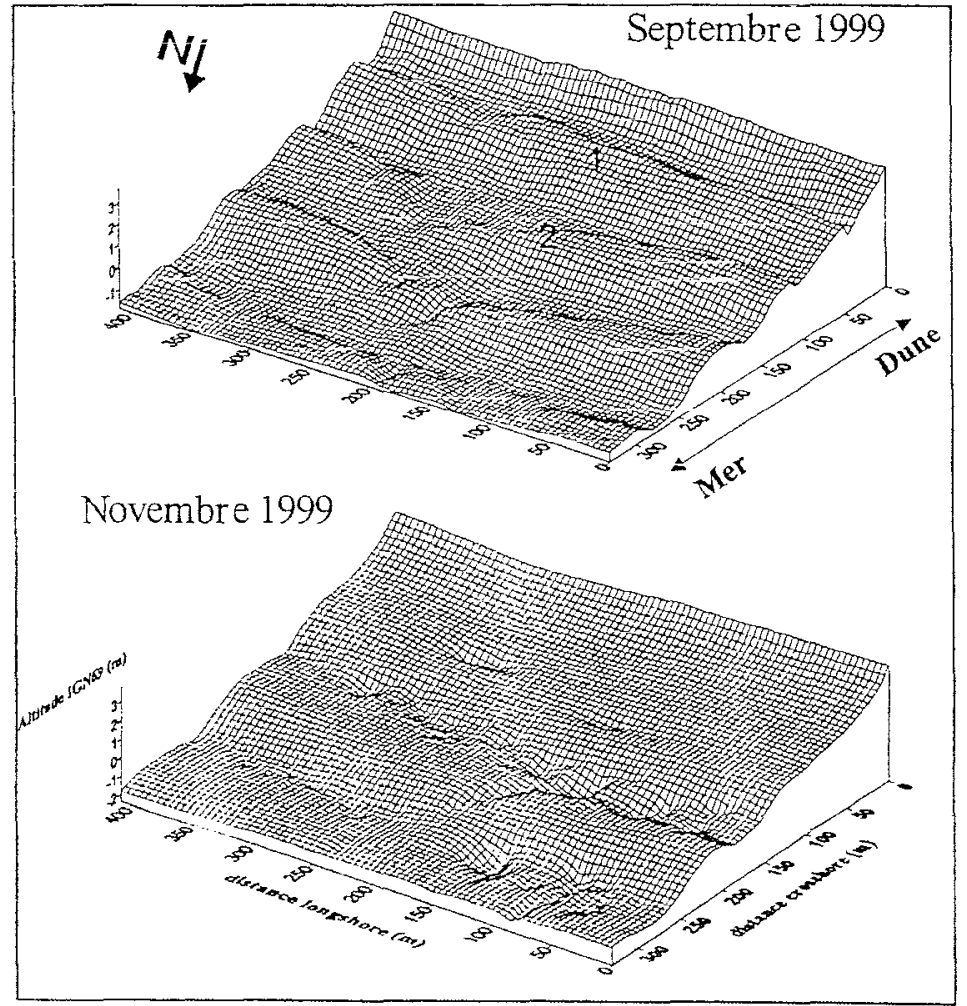

Figure 4 - Modèles Numériques de Terrain MNT - 3D

Figure 4-3D Digital Elevation Models 
L'évolution morphologique est marquée par la disparition de la première barre sur le haut estran et par une régularisation de la topographie générale de la plage. Le profil a tendance à prendre une forme concave. Sur la moyenne et la basse plage, la position des barres reste stable mais les bâches ont tendance à se combler.

\section{Bilan sédimentaire}

La réalisation de Modèles Numériques de Terrain permet le calcul du volume du stock sédimentaire de la plage et l'évaluation de ses variations. Le niveau de référence pour les calculs est représenté par l'isoligne - $3 \mathrm{~m} \mathrm{IGN}_{69}$; il est inférieur à l'altitude minimale atteinte par les basses mers au cours des levés de l'année 1999. Les volumes calculés sont soumis à une incertitude de $+/-2700 \mathrm{~m}^{3}$, correspondant à une imprécision de $+/-2 \mathrm{~cm}$ sur la mesure de l'altitude des points topographiques au DGPS.

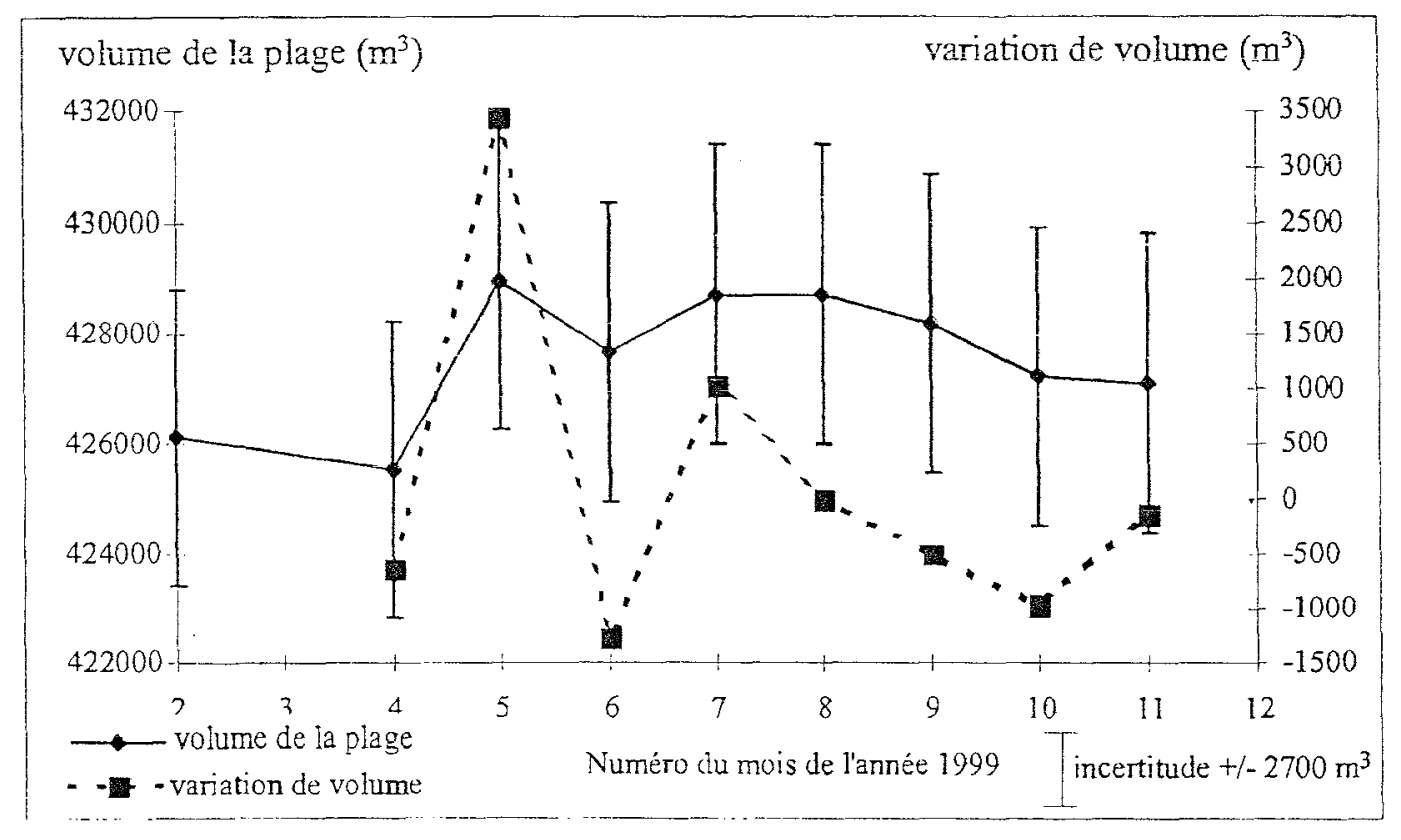

Figure 5 - Evolution du stock sableux de la plage au cours de l'année 1999 Figure 5 - Volumetric evaluation of sediment budget of the beach during 1999

Au cours de la période d'étude, l'amplitude des variations du volume sédimentaire de la plage d'Omaha-Beach se situe, pour la plupart des levés, dans l'incertitude de la mesure (figure 5). Le pourcentage de sable mobile varie entre $-0,5 \%$ dans le cas de pertes et $+0,8 \%$ pour la variation la plus significative $\left(+3432 \mathrm{~m}^{3}\right)$, observée entre avril et mai 1999. Ces caractéristiques semblent indiquer que la réorganisation morphologique de la plage se ferait à volume constant. Les fuites ou les apports résiduels nets de sable entre la zone subtidale et la plage intertidale etou par dérive littorale seraient donc limités. 


\section{Le contexte hydrodynamique et ses implications dynamiques}

L'enregistrement des caractéristiques de l'agitation $\left(\mathrm{H}_{s}\right.$ et angle d'incidence) au cours de l'année 1999 est représenté sur la figure 7. Les dates des levés topographiques présentés précédemment y sont également reportés. Les conditions de houle incidente au cours des trois phases d'évolution morphologique de la plage précédemment décrites sont les suivantes :

- Durant la phase de construction et de migration des barres vers le haut de plage (février - juillet 1999), les conditions hydrodynamiques sont dominées par une agitation faible bien que deux courtes périodes de tempête se produisent. L'impact de ces périodes de haute énergie, où les hauteurs atteignent $3 \mathrm{~m}$, semble réduit sur l'évolution morphologique globale des systèmes de barres à l'échelle temporelle du suivi. Néanmoins, ces deux événements de haute énergie pourraient être responsables de l'incision des chenaux de drainage dont la position reste stable de mai à juillet 1999. L'afflux d'eau au niveau de la haute plage en phase de tempête entraîne un courant de compensation vers le large qui inciserait les barres à des endroits préférentiels, l'érosion de ces chenaux s'accentuant pendant la phase descendante de la marée en liaison avec la vidange des bâches.

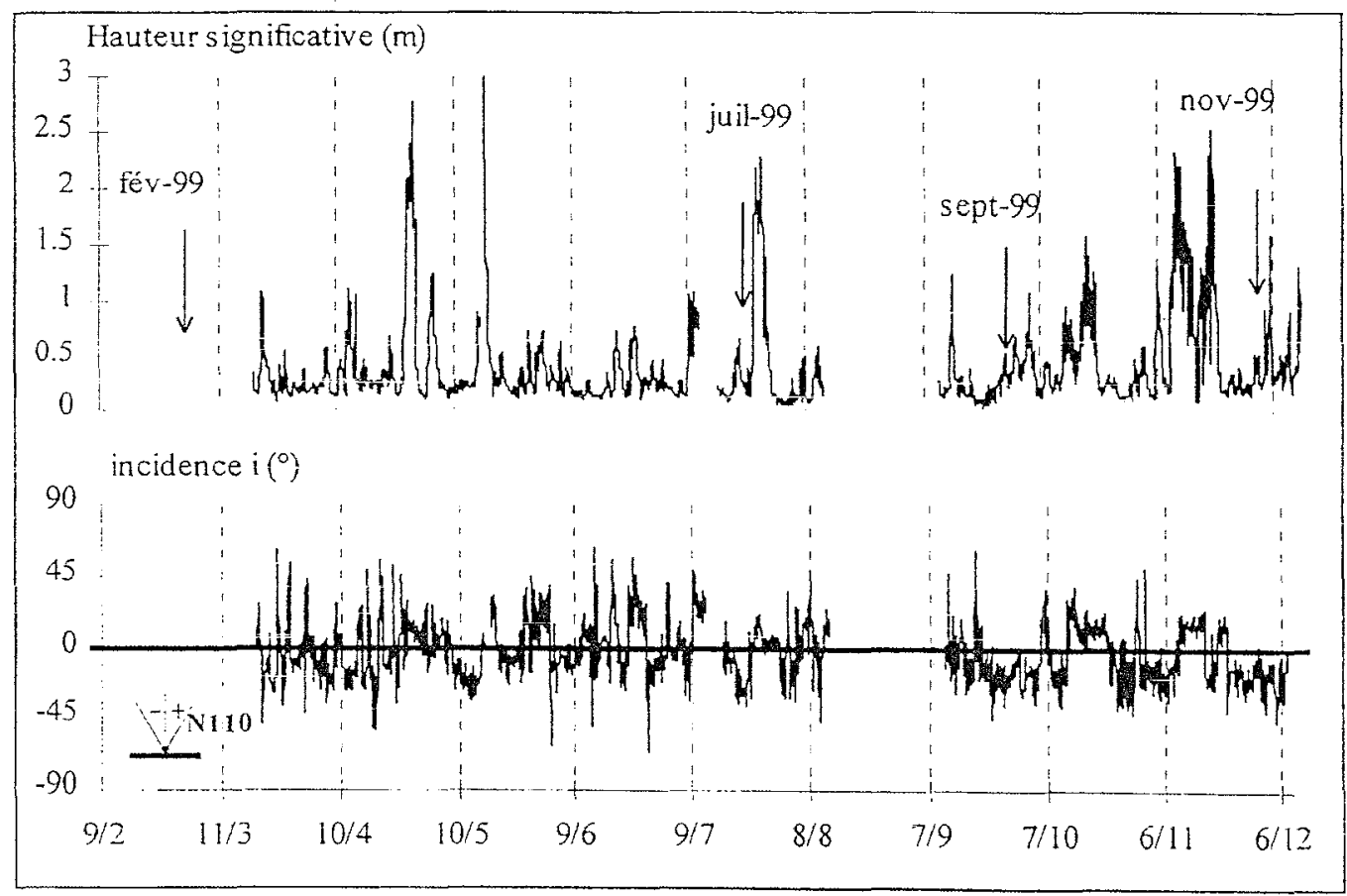

Figure 7 - Conditions hydrodynamiques enregistrées en bas de plage au cours de l'année 1999

Figure 7 - Hydrodynamic conditions recorded on the lower part of the beach during 1999 
- La période de migration latérale des chenaux de drainage de juillet à septembre 1999 souffre d'un manque de données hydrodynamiques en raison d'un dysfonctionnement technique de l'instrumentation. Néanmoins, l'examen des données météorologiques enregistrées au sémaphore de Port-en Bessin (METEOFRANCE) montre que, durant la période de juillet à août $1999,65 \%$ des vents de mers proviennent du secteur Est avec des vitesses pouvant atteindre 16 $\mathrm{ms}^{-1}$. Ce contexte génère une agitation importante et des transports sableux par dérive littorale Est-Ouest, dont l'intensité reste à évaluer notamment par des expérimentations de traçage fluorescent. Ce transport longitudinal en tempête pourrait être responsable de la migration des chenaux vers l'Ouest.

- La période automnale est dominée par des houles de plus forte amplitude. Les tempêtes, pendant lesquelles les hauteurs significatives dépassent $2 \mathrm{~m}$ durant plusieurs jours, sont responsables de la destruction des barres de la haute plage.

Les séquences de mobilité des barres mises en évidence confirment les résultats des études entrenrises sur ce type de morphologies, à une échelle de temps comparable (Mulrennan, 1992, Levoy et al., 1998). Les résultats présentés élargissent l'approche morphodynamique en tenant compte de la migration latérale des chenaux de drainage (effet 3D).

\section{Conclusions}

Au vu des résultats obtenus au cours de l'année 1999, l'évolution morphologique de la plage d'Omaha-Beach apparaît étroitement liée aux caractéristiques de l'agitation incidente. Deux modes d'évolution morphologique ont pu être identifiés. La prédominance de faible houle entraîne une migration et une accrétion des barres vers le haut de plage. La succession de tempêtes automnales a, par contre, un effet destructif sur la partie haute de la plage et plus limité sur le moyen et bas estran. Un comportement morphodynamique très différent des diverses parties de la plage et de leurs barres sableuses associées est mis en évidence. L'occurrence d'événements de haute énergie isolés semble ne pas avoir d'impact direct sur un système de barres bien individualisées. Cependant, ils semblent responsables de la dynamique des chenaux perpendiculaires aux barres, en régissant notamment leur apparition sur l'estran. La poursuite des levés topographiques réguliers durant l'année 2000 va compléter la base de donnée acquise afin de réaliser une analyse poussée de la réponse morphologique aux forçages hydrodynamiques. Ce travail doit s'appuyer sur une paramétrisation précise des conditions hydrodynamiques et de la topographie en intégrant la disparité morphodynamique de l'estran. 


\section{Références}

KING C.A.M., \& WILLIAMS W.E., 1949. The formation and mouvement of sand bars by wave action. Geographical Journal, 113, p.70-85.

LEVOY F., ANTHONY E., BARUSSEAU J.P., HOWA H., \& TESSIER B., 1998. Morphodynamique d'une plage macrotidale à barres. Comptes Rendus de l'Académie des Sciences (Paris), 327, p.811-818.

MULRENNAN M.E, 1992. Ridge and Runnel Beach Morphodynamics: An Example from Central East Coast of Ireland. Journal of Coastal Research, 8(4), p.906-918.

ORFORD J.D., \& WRIGHT P., 1978. What's in a name ? Descriptive or genetic implications of « ridge and runnel» topography. Marine Geology, 28, M1-M8.

\section{$\underline{\text { Remerciements }}$}

Cette étude est effectué dans le cadre du Programme National Environnement Côtier - ART7 «Dynamique sédimentaire ». Les auteurs remercient de plus METEOFRANCE pour les données météorologiques ainsi que toutes les personnes qui ont participé aux mesures sur le terrain. 